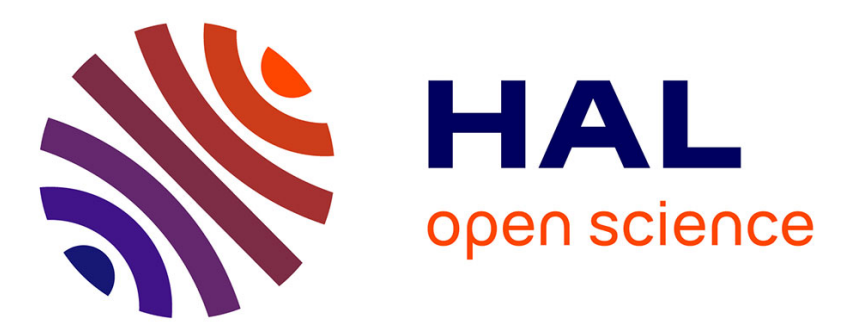

\title{
Premiers résultats du Recensement de 1962 dans les Pyrénées et les régions sous-pyrénéennes
}

\author{
Georges Bertrand
}

\section{To cite this version:}

Georges Bertrand. Premiers résultats du Recensement de 1962 dans les Pyrénées et les régions souspyrénéennes. Revue Géographique des Pyrénées et du Sud-Ouest, 1963, 34 (1), pp.49-64. 10.3406/rgpso.1963.4756 . hal-02615407

\section{HAL Id: hal-02615407 \\ https://hal-univ-tlse2.archives-ouvertes.fr/hal-02615407}

Submitted on 22 May 2020

HAL is a multi-disciplinary open access archive for the deposit and dissemination of scientific research documents, whether they are published or not. The documents may come from teaching and research institutions in France or abroad, or from public or private research centers.
L'archive ouverte pluridisciplinaire HAL, est destinée au dépôt et à la diffusion de documents scientifiques de niveau recherche, publiés ou non, émanant des établissements d'enseignement et de recherche français ou étrangers, des laboratoires publics ou privés. 


\section{Premiers résultats du Recensement de 1962 dans les Pyrénées et} les régions sous-pyrénéennes

\section{Georges Bertrand}

\section{Citer ce document / Cite this document :}

Bertrand Georges. Premiers résultats du Recensement de 1962 dans les Pyrénées et les régions sous-pyrénéennes. In: Revue géographique des Pyrénées et du Sud-Ouest, tome 34, fascicule 1, 1963. pp. 49-64;

doi : https://doi.org/10.3406/rgpso.1963.4756

https://www.persee.fr/doc/rgpso_0035-3221_1963_num_34_1_4756

Fichier pdf généré le 05/04/2018 


\section{I S E A U P O I N T \\ PREMIERS RESTITATS DE RECENSEMENT DE 1962 DANS IAS PYRENEES WT LES REGIONS SOLS-PYRENENNES}

Les premiers dépouillements du Recensement de la population de 1962 n'apportent guère de surprises (1). Dans leurs grandes lignes. ils confirment les tendances relevees dans les trois recensements précédents et les prévisions de divers spécialistes (2). La situation démographique d'ensemble ne s'est done pas améliorée. Les Pyrénées continuent a perdre des habitants. Cependant, l'evolution est loin d'etre uniforme et l'exode n'est pas généralisé.

1. Les tendances générales (tableau l) (3). De 195.t à 1962. la population des six départements pyrénéens et sous-pyrénéens (Pyré-

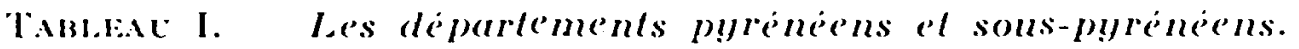

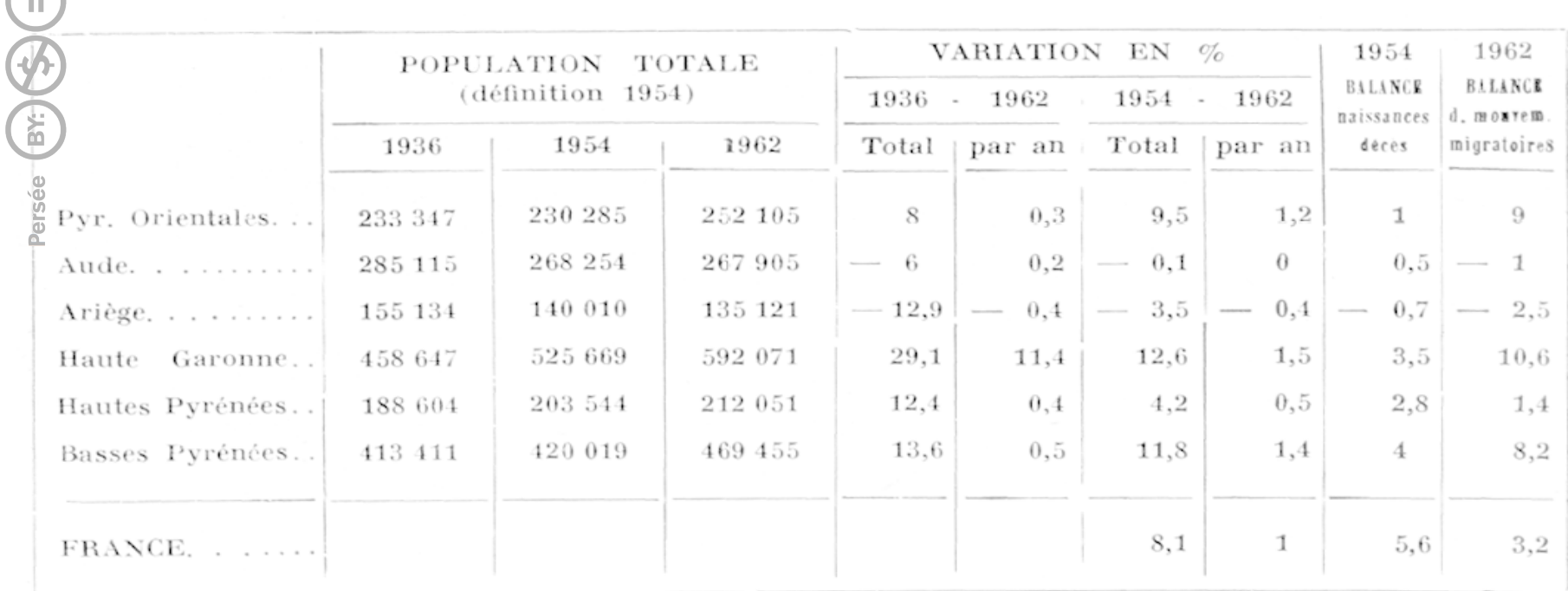

i) cefte mise at point nat pas dautre ambition que de presenter quelefues

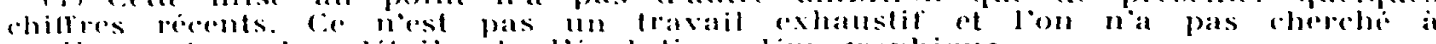
copliquer folls les details de levolution demographigue.

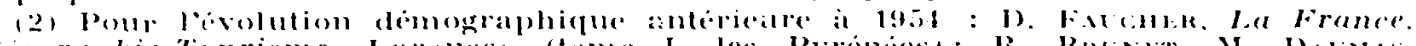

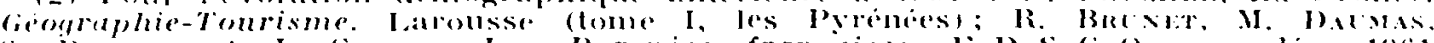

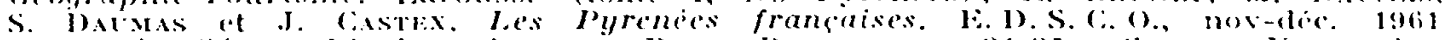

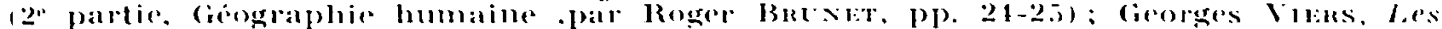

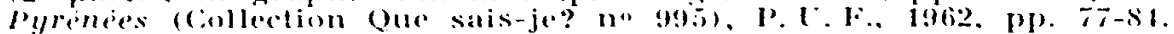

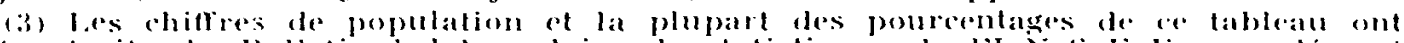

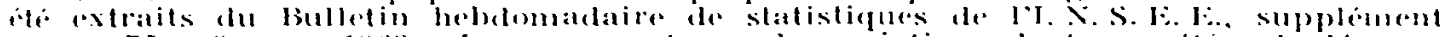

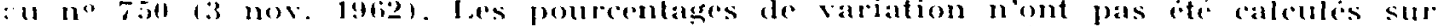

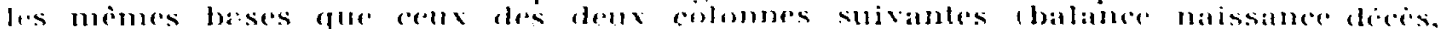

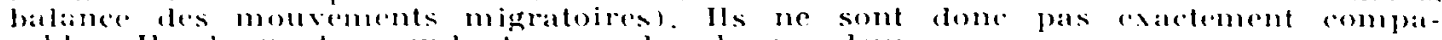
mables. Ils domment cependant un ordere de srandear. 
nées-Orientales, Aude, Ariège, Haute-Garonne, Hautes-l'yrénées, Basses-Pyrénées) est passée de 1787700 habitants à 1928700 habltants, soit une augmentation de $7 \%$ (France $8 \%$ ). La Haute-(iaronne, les Basses-Pyrénées et les Pyrénées-Orientales, qui s'étalent largement hors de la chaine et qui possèdent de grandes agglomérations, dépassent la moyenne nationale avec $12,6 \%, 11,8 \%$ et $9,50 \%$. Par contre, la population ariégeoise a diminué de $3,5 \%$ (perte de 4889 habitants). Pendant la même période, l'excédent des naissances sur les décès est partout faible : Basses-Pyrénées $4 \%$ (16000 personnes), Haute-Garonne 3,5\% (18 100), Hautes-Pyrénées 2,8\% (5 400), Pyrénées-Orientales $1 \%$ (2 300). La balance naissances-dlécès, en équilibre dans l'Aude, est négative en Ariège avec - . $0,70(1000)$, la moyenne française étant de $+5,6 \%$. Entre les deux derniers recensements, l'immigration a fourni un gros appoint à certains départements. La Haute-Garonne a gagné 54000 habitants, les Basses-Pyrénés 33500 et les Pyrénées-Orientales 20 100. Au contraire, l'Ariége et l'Aude ont perfu par émigration respectivement 3400 et 2500 habitants. Mais tous ces chiffres sont actuellement gontlés par l'installation massive des populations algériennes. De toute manière, ces résultats n'ont qu'une signification limitée. Le cadre départemental masque de profoncles divergences d'evolution. En effet, si les villes des basses vallées et, surtout, des régions sous-pyrénénnes, s'accroissent dans des proportions parfois considérables, les communautés agropastorales des hautes vallées et des soulanes escarpées s'effondrent irrémédiablement.

Pour les seuls arrondissements prénéns (tabloau II), la population a diminué d'environ $5 \%$ entre 1954 et 1962 : $8 \%$ pour l'arron-

Thmbac II. Types d'arrondissements pyrine'ns.

\begin{tabular}{|c|c|c|c|c|}
\hline & & 1954 & 1962 & Différ \% \\
\hline Pyrénćes Orientales.. & Prades. . . . . . . & 37133 & 36702 & -1 \\
\hline Aude. . . ... & Limoux. . . . . . . & 47964 & 45819 & -4 \\
\hline Ariège. . . ....... & Foix. . ......... & 50138 & 49379 & -2 \\
\hline \multirow{3}{*}{ Pyrénées.... } & Saint-Girons. . .... & 35462 & 32403 & -8 \\
\hline & Argelès-Gazost. . . . & 42032 & 40844 & -3 \\
\hline & Bagnères de Bigorre. & 16331 & 15855 & -3 \\
\hline
\end{tabular}

dissement de Saint-(iirons, tombe de 35462 habitants à 32403 habitants, $4 \%$ pour celui de Limoux, $3 \%$ pour ceux d'Argeles-ciazost et de Bagnères-de-Bigorre (4). L'évolution des cantons est encore plus

(1) Les pourcentages sont domis aves une marge d'erreur possible de $1 \%$. lEn efret il nat pas eté temu comple des decimales. Par conte nous navons pas cru devoir supprimer les décimales des chimres fournis par l'I. N. S. F. E. 
significative (tableau III). Les cantons des hautes vallées, surtout à l'Est et au centre de la chaine, ont perdu entre 10 et 30 \% de leur population entre 1954 et 1962 . Le canton de Quérigut, très forestier et dépourvu d'industries, avait 1250 habilants en 1936,1078 en 1954. Il n'en reste que 694 en 1962 . De 1954 à 1962 , Vielle-ture a perdu $22 \%$ de ses effectifs. De lels cfrondrements sont moins fréquents à

Tambia III. Qnelques types de cantons.

\begin{tabular}{|c|c|c|c|c|}
\hline & 1936 & 1954 & 1962 (1) & $1954-1962$ \\
\hline HAUTES VALLEES & & & & \\
\hline Quérigut (A.). . . . . . . . . . & 1250 & 1078 & 694 & $-36 \%$ \\
\hline Luz-Saint Sauveur (H.-P.) ..... & 4281 & 6113 & 4037 & $-34 \%$ \\
\hline Massat (A.) . . . . . . . . . & 6784 & 3591 & 2464 & $-32 \%$ \\
\hline 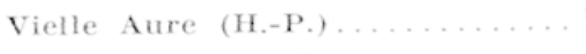 & 2182 & 2460 & 1934 & $-22 \%$ \\
\hline Mont-Louis (P.-O.) ....... & 3687 & 3166 & 2520 & $-21 \%$ \\
\hline Castillon (A.). ............ & 8063 & 5459 & 4409 & $-20 \%$ \\
\hline Oust (A.). . . . . . . & 7724 & 4557 & 3929 & $-16 \%$ \\
\hline Aucun $(\mathrm{H},-\mathrm{P}),. \ldots \ldots \ldots \ldots$ & 3277 & 3054 & 2641 & $-14 \%$ \\
\hline Prat-de-Mollo (P.-O.). . . . . . & 6215 & 5032 & 4376 & $-13 \%$ \\
\hline Campan (H.-P.) . . . . . & 3726 & 3708 & 3376 & $-9 \%$ \\
\hline Tardets (B.-P.) . . . . . . . . & 6816 & 5772 & 5276 & $-9 \%$ \\
\hline Accous $\left(B_{-}-P_{1}\right) \ldots \ldots \ldots$ & 5662 & 4428 & 4121 & $-7 \%$ \\
\hline Laruns (B.-P.). . . . . . . . . & 4285 & 4037 & 3873 & - $4 \%$ \\
\hline $\begin{array}{c}\text { REGIONS FREPYRENEENNES } \\
\text { ET SOUS-PYRENEENNES }\end{array}$ & & & & \\
\hline Lannemezan (H.-P.). . . . . . . . & 7782 & 9994 & 11486 & $+15 \%$ \\
\hline Argelès-sur-Mer (P.-O, . . . . . . . . & 21202 & 21448 & 23772 & $+8 \%$ \\
\hline Céret $($ P.-O.) . . . . . . . . . & 11922 & 11755 & 12511 & $+7 \%$ \\
\hline Lavelanet (A.). . ................ & 12013 & 13668 & 13967 & $+2 \%$ \\
\hline Prades (P.-O.). . . . . . . . . & 11525 & 12033 & 12325 & $+2 \%$ \\
\hline Saint-P'alais (B.-P.) . . & 11037 & 9832 & 9426 & $-4 \%$ \\
\hline Le Mas d'Azil (A.) . . . . . . . & 5650 & 4917 & 4606 & $-6 \%$ \\
\hline
\end{tabular}

lextericur de la haute montagne. Par exemple, la population des Basses-Pyronees resiste un peu mieux : pertes de 5 \% a Saint-Etiennede-bä̈gory et de 9 y a Tardets. Les cantons sous-pyreneens gagnent en general des habitants : Lannemezan (!- 15 \% (cert $\left(-7 C_{c}\right)$. I.es Prépyrénés occupent une situation intermédiaire. Quand elles sont industrialisees et que les industries sont prosperes, clles participent 
a laccroissement des régions sous-pyrénéennes : lavelanet $(+2 \%)$. Sans industries, elles ne sont pas plus favorisées que la haute montagne : Le Mas d'Azil $(-\ldots 6 \%)$. Ia haute montagne se vide, plus vite dans le secteur méditerranéen et ariégeois que dans le secteur central at surtout que dans les Pyrénées basques et béarnaises. La moyenne montagne conserve un peu mieux ses habitants si elle est industrialisée. L'essentiel de l'accroissement se situe hors de la chaine et plus particulièrement dans trois secteurs : la région sous-pyrénéenne centrale entre la ciaronne et le gave d'Oloron, le littoral basque et le Roussillon. C'est, avant tout, une croissance de type urbain. L'évolulion récente revêt done un double aspect : opposition entre la monlagne et ses bordures, opposition entre population rurale et population urbaine.

2. Persistance, et parfois accélération de l'exode rural. a. Quelque's siluations critiques. I, célèbre canton de Massat (Couserans) nous en donne une premiere et remarquable démonstration (tableau IV) (j). Aucune inclustrie. aucune amélioration récente de léconomie pasiorale pour retenir les montagnards, isolés dans le bassin de l'Arac

TABlate IV. Le canton de hassal.

\begin{tabular}{|c|c|c|c|c|c|c|c|}
\hline & 1936 & 1954 & 1962 & $\begin{array}{r}\text { PE } \\
\text { To7 } \\
1936\end{array}$ & $\begin{array}{l}\text { ES } \\
\text { ES } \\
1962\end{array}$ & $\begin{array}{c}\text { PERTES } \\
\text { ANNUELLES } \\
1936-1962\end{array}$ & $\begin{array}{c}\text { CHANCRs } \\
\text { approximatives } \\
\text { de surrie (5) }\end{array}$ \\
\hline Biert. . ........... & 1515 & 512 & 365 & 1150 & $73 \%$ & 44 & 8 :ns? \\
\hline Le Port. . ...... & 910 & 448 & 282 & 628 & $69 \%$ & 24 & 12 ans? \\
\hline Boussenac. . .... & 1171 & 737 & 398 & 773 & $65 \%$ & 30 & 13 ans? \\
\hline Aleu. . . . . . . & 516 & 293 & 196 & 320 & $61 \%$ & 12 & 16 ans? \\
\hline Soulan. . ......... & 922 & 544 & 403 & 519 & $56 \%$ & 20 & 21 ans? \\
\hline Massat (commune). & 1750 & 1057 & 820 & 930 & $47 \%$ & 36 & 22 ans? \\
\hline Canton. . . . . . & 6784 & 3591 & 2464 & 4320 & $64 \%$ & 166 & \\
\hline
\end{tabular}

(600) a $900 \mathrm{~m}$ dialtitude entro les massifs de lidrize et des TroisSeigneurs. Lomigration est une tradition bien etablie (6). Le canion a perdu 4320 habitants de 1936 a 1962 ; il ne reste plus que 2464 habitants. Le rethme annuel de depopulation est denviron 4 \%e, soit 160 a 170 habitants en moins. Si. hypothèse probable, ce rythone se

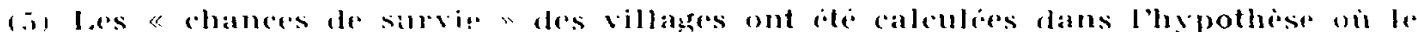
Fithe actuel de depelubenent se maintiendrait.

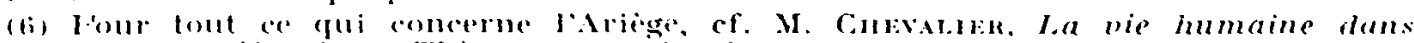

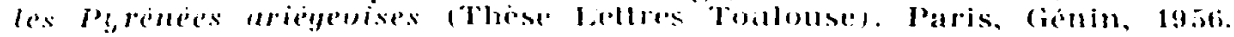


maintient, les villages seront abandonnés dans une vingtaine d'annires. Certaines communes : Biert, le Port, Boussenac, ne compteront plus l'habitants d'ici une dizaine d'années. Tout effort d'aménagement eventucl est dejà compromis d'avance. Or, la dépopulation paraît s'accélerer à mesure que le nombre d'habitants diminue. C'est ce que montre le diagramme des communes des Pyréaées-Orientales de moins de 100 habitants (fig. 1). En 8 ans, de 1954 à 1962, les 48 communes considérées ont évolué de la façon suivante : 14 communes ent perdu plus du quart de leurs habitants deux dentre clles plus

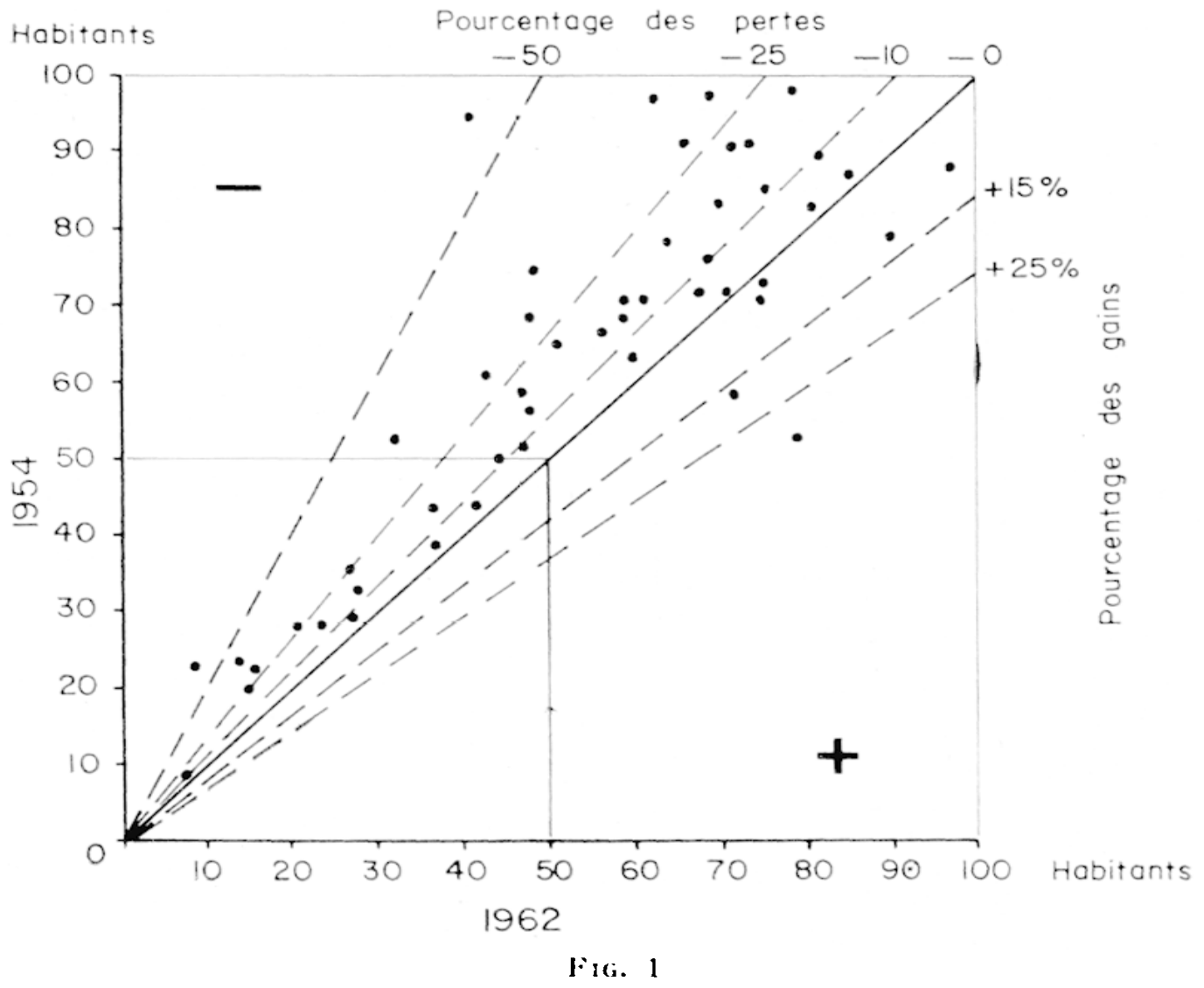

lieffondrement da la population dans les commu:ss de moins de 100 habitants (1954-1962).

de la moitie); 20 communes entre 10 et $24 \% ; 10$ communes entre 1 et 9 \% : 6 seulement ont augmente, dont 2 de plus de $15 \%$. Parmi ces « communes de poche » citons (iloriane (16 hab.), Sansa (15 hab.); enfin Caudies, qui ne compte plus que 8 habitants el qui en avait 69 en 1936! Que restera-t-il au moment du prochain recensement?

b. L'évolution mancée de la vallée pyrénéenne de l'Ariege (fig. 2). la situation démographique $y$ est critique : emigration définitive. 
vieillissement de la population active, mariages très rares, dénatalité (7). Cependant la diminution n'est pas uniforme. Les petits villages des hautes soulanes (Tabe, Vicdessos, Trois-Seigneurs) et des vallées secondaires (Haute-Barguillère, vallée de Rabat, Vicdessos, rallee de l'Oriège) sont durement touchés. Ce sont sans exception

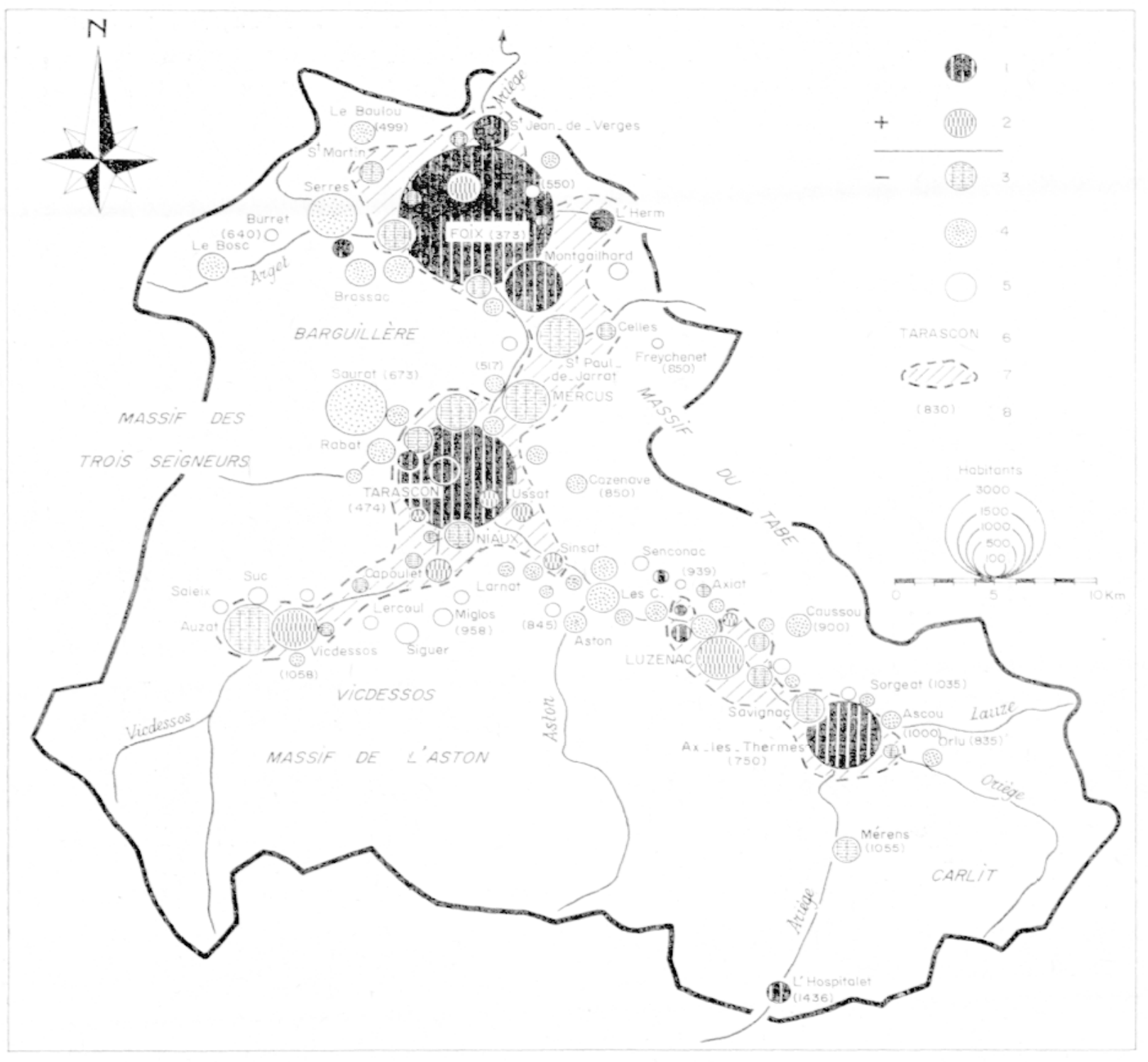

Fig. 2

L'évolution de la population dans la vallée pyrénéenne de l'A riège (1954-1962).

1. Gain super:eur a $10 \%$. 2. Gain inférieur a $10 \%$. Pertes inférieures; a $10 \%$. - 4. Entre 10 et $20 \%$ de pertes. - 5. Plus de $20 \%$ de pertes. - 6. Centres industriels (ajouter Auzat). - 7. Secteurs les moins défarorisés. - 8. Altitude des villages.

(7) Georges BERTuxv, le déclin dimographique des hautes valleses pyrinéennes: l'exemple du canton de's Cabannes (Ariege). Rev. géogr. P’yénées Sud-Ouest, 1959, pp. 82-87, 2 fig. 
des communautés agro-pastorales qui doivent subsister en dépit de l'exiguïté des parcelles, de l'eloignement des estives et de la mauvaise commercialisation des produits de l'élevage. De 1954 à 1962 , les villages accrochés aux grands versants, entre $750 \mathrm{~m}$ et $1200 \mathrm{~m}$ d'altitude, ont perdu plus du ticrs, ou plus de la moitié, de leur population. Dans le Vicdessos, a Lercoul (13 habitants en 1962) la perte atteint $55 \%$ a Saleix (31 habitants) $48 \%$. Freschenet et Appy, sur les pentes du Tabe, ont pertu $43 \%$ \% et $38 \%$ de leurs effectifs. Iarcat, sur l'Aston, est tombe de 101 a 63 habitants. Deux causes en sont responsables : léclatement du système agro-pastoral traditionnel et lisolement. Il ne parait pas $y$ avoir de remedes. La population tend a se concentrer au fond de la basse vallec autour des petils centres industriels. Dimportants aceroissements se rencontrent autour de Tamascon (usines d'électrochimie et d'electrométallurgie de Sabart, Niaux ef Mereus). De 1954 a 1962 , Taraseon s'est gonfle de 12 \% che 3184 a 3543 habitants). Ia construction de logements $y$ fait de grands progris ef l'equipement commereial se complete. Banat a gagne $21 \%$. Quie $14 \%$. Issat $1 \%$. ote. re chef-lieu du departement. Foix, a progressé de $15 \%$ (7 (632 à 8770 habitants), pourcentage dépassé par la commune limitrophe de Montsallarel de plus en plus rattachée a lagglomération fuxéenne of cui détient le record de la vallée montagnarde de lidrioge arec $47 \%$ (667 a 979 habitants). Ice troisieme noyau d'aceroissement, moins dendu, entoure l'usine des Tales de Iuzenac : I'rs et Lassur $(14 \%)$. Vernaux et Iuzenac $(8 \%)$. Laugmentation de la population des centres isolés est due soit a l'électrochimie (Viclessos : de 563 a 613 habitants). soit au thermalisme et au tourisme (Ax-les-Thermes : $11 \%$, lssat-les-13ains : $1 \%$ co), soil alux grands chantiers de l'E. I). F. gui gonflent artifieiellement la population du village de l'fospitalet $(37 \%)$. Au moins temporairement, l'industrie participe au maintien des montagnards en ajoutant les salaires aux revenus de l'élevage. Te genre de vie mixte est assez repandu. Les communes agro-pastorales du Val d'Ariege, ou le nombre des ouvriers eleveurs est fable, perdent en movenne de 10 at $20 \%$ de leurs habitants (Vebre, Verdun, Iston dans le canton des Cabannes). (cette évolution nuancée suggere phusicurs remareques. En premier lieu, la perte de population est en gros proportionnelle à la taille du village. Les communes de moins de 100 habilants sont menacées de disparition (Lercoul, Orus dans le Vicdessos, Burret en Barguillere, Bestiac sur la soalane du Tabe). On rejoint le cas. précedemment envisagé, des petits villages des Pyrénées-Orientales. En sccond lieu. toutes les communes agro-pastorales ont perdu entre 10 et $40 \%$ de leurs habitants. Rien ne semble plus pouvoir freiner leur décadence. Par contre l'industric et, dans une moindre mesure, le thermalisme permettent à la population de se maintenir ou même de s'aceroître. La grande industrie moderne joue un rôle considérable comme le souligne l'extension récente de Tarascon. I'usine des Talcs de Luzenac influe moins sur la population car clle ofre surtout des 
travaux pénibles (carrière de Trimouns a $1400 \mathrm{~m}$ d'altitude) que les montagnards ne disputent pas à la main-d'euvre saisonnière espagnole et portugaise. Une population de type nouveau, plus ouvric̀re que paysanne, se développe dans les bourgs et les villages des basses vallées. Les montagnards purs tendent à n'être plus qu'une minorité. Le genre de vie mixte se maintient rarement après la deuxième génération. La montagne est de moins en moins exploitée. Sur les 32500 habitants que contient la vallée pyrénéenne de l'Ariège. 13877. soit $42 \%$, sont groupés a Foix, Tarascon et Ax-les-Thermes. La population se concentre de plus en plus dans les villes. Mais les bourgs ne participent pas à cet essor : Les Cabannes a perdu $11 \%$ de se's habitants entre 1954 et 1962 .

3. La crise des villages-centres et des bourgs. -... C'est un aspect particulièrement grave du dépeuplement, à la fois conséquence et cause de l'exode rural. I.es trois-quarts des bourgs et des villages-centres de la zone montagneuse se vident de leurs habitants et certains retournent à l'état de simples villages à équipement commercial très réduit. Cette évolution est particulièrement nette dans les hautes vallées. De 1954 à 1962 . Ies populations de Luz-Saint Sauveur, Vielle Aure, Massat et Mont-Louis se sont effondrées entre 17 et $40 \%$. En moyenne. les pertes sont de 4 a $16 \%$. La plupart de ces petits centres méritent a peine le qualificatif de commercial tant leur équipement est médiocre: Durban et Tuchan dans les Corbières, Belcaire en pays de Sault, Axat et Couiza dans la vallée de l'Aude, les Cabannes dans le Val d'Ariege, Arreau en vallée d'Aure. Prenons par exemple le village-centre de Quérigut. Il ne résiste pas mieux que les autres villages du Donnezan. De 1954 à 1962, l'ensemble du canton a diminué de $36 \%$, le village-centre a perdu $33 \%$, (267 à 178 habitants). I'équipement commercial se réduit à quelques commerces alimentaires. I.es habitants des six autres villages du Donnezan sont donc complètement isolés. Ie moindre achat nécessite un long déplacement à Quillan. En effel, Axat et Couiza, plus proches, ne sont guère mieux équipés que Quérigut. Les bourgs et, à plus forte raison les villages-centres, qui sont restés uniquement ruraux sont donc en pleine décadence.

Le tourisme et le thermalisme n'apportent pas toujours les anéliorations escomptées. surtout dans les petits villages (tableau V) (8). Les Eaux-Bonnes et Barèges ne semblent pas avoir progressé. Cauterets a perdu au moins $10 \%$ de ses habitants. Saint-Lary. qui s'équipe activement pour les sports d'hiver, a réalisé quelques gains, ainsi d'ailleurs que la station thermale de Barbazan. Ax-les-Thermes, qui a progressé de $10 \%$ entre 1954 et 1962 , donne une idée de laction positive, mais limitée, que peut exercer une petite station thermale et

(8) Voir note 11. In présence dans beaucoup de ces petits centres d'une importante population mobile (grands chantiers de l'E. $D$. F. par exemple) ne permet pas de domer des chifres précis. 
TaBhac V. Evolution de quelques communes possedant une station thermale on whe station de sports d'hiver.

\begin{tabular}{|c|c|c|c|c|}
\hline & 1936 & 1954 & 1962 & $1954-1962$ \\
\hline Barbazan (H.-G.). . . . . . & 421 & 438 & 511 & $+16 \%$ \\
\hline Ax-les-Thermes (A.). . . . . & 1321 & 1422 & 1564 & $+10 \%$ \\
\hline 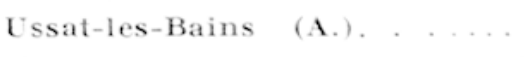 & 131 & 170 & 172 & $+1 \%$ \\
\hline Saint-Lary (H.-P.). . . . . . & 282 & 633 & 529 & $-17 \%$ \\
\hline Capvern (H.-P.). . . . . . & 872 & 925 & 754 & $-19 \%$ \\
\hline Cauterets (H.-P.) . . . . . . & 1253 & 1242 & 989 & $-20 \%$ \\
\hline Barèges (H.-P.) . . . . . . . & 143 & 374 & 265 & $-30 \%$ \\
\hline Aulus (A.). . . . . . . . & 590 & 402 & 277 & $-31 \%$ \\
\hline Eaux-Bonnes (B.-P.). . . . . & 462 & 558 & 375 & $-33 \%$ \\
\hline
\end{tabular}

touristique sur les villages voisins. Les montagnards bénéficient de fout l'équipement «tertiaire" de la station : commerces variés et bien approvisionnés, médecins, elc. Ils y trouvent parfois un emploi. soit permanent, soit saisonnicr. Cependant la portéc de ce phénoméne ne doit pas etre exagéré. Autour d'Ax-les-Thermes. le dépeuplement continue.

L'implantation dindustries en milieu rural exerce une action bien plus efficace. Depuis l'exploitation du gaz de Saint-Marcet, en 1939, la cluse de Boussens (9), qui possédait déjà de petite's usines, s'est

Tableac VI. .. Ia cluse de Bollssens.

\begin{tabular}{|c|c|c|c|c|}
\hline BOURGS ET VIILAGES IN- & 1936 & 1954 & 1962 & $1954-1962$ \\
\hline Boussens. . . ........ & 400 & 520 & 707 & $+31 \%$ \\
\hline Roquefort sur Garonne... & 544 & 695 & 860 & $+24 \%$ \\
\hline Palaminy. . ........... & 504 & 500 & 521 & $+2 \%$ \\
\hline Martres-Tolosane. . . ..... & 1266 & 1809 & 1811 & - \\
\hline $\begin{array}{l}\text { VILIAGES DES PETITES } \\
\text { PYRENEES }\end{array}$ & & & & \\
\hline Ausseing. . . . . . . . . & 127 & 80 & 72 & $-10 \%$ \\
\hline Belbèze. . . . . . . . . . & 253 & 213 & 174 & $-18 \%$ \\
\hline 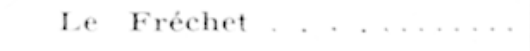 & 97 & 107 & 84 & $-22 \%$ \\
\hline Marignac. . . . . . . . & 178 & 186 & 138 & $-26 \%$ \\
\hline
\end{tabular}

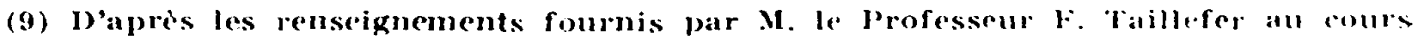
dune excursion de I'Institut de Geographie de "roulouse. 
fortement industrialisée (usine de dégazolinage, puis cimenterie Lafarge). Les façades claires des cités ouvrières tranchent au milieu des vieux villages patinés. Laccroissement de population porte sur quatre communes (tableau VI) : Boussens qui a augmenté de $31 \%$ entre 1954 et 1962 ; Roquefort-sur-(iaronne $(24 \%)$ qui est devenu, avec 860 habitants, un bourg très actif; enfin, Palaminy et MartresTolosane (1266 habitants en 1936. $1811 \mathrm{en} \mathrm{1962).}$

cet accroissement reste exceptionnel et ne doit pas masquer la disparition définitive du petit équipement commercial et du secteur tertiaire dans la plupart des rallees preneennes. Iofenondrement des bourgs et des villages-centres qui participent étroitement a la vie sociale et à l'economie des monlagnes ne peut que favoriser l'exode rural. Il n'y a plus de centre intermérliaire entre le village qui se depeuple el la ville qui grandit.

4. Les progrès des villes. - I'expansion urbaine, surtout nelte apròs 1954, donne un bon rang aux départements pyrénéens et cache la decadence des communatés rurales. Cependant, cest par excellence un phénomine sous-prénéen. En effet a peine troure-ton une quinzaine de villes de taille modleste à l'intérieur de la chaîne (Foix, Bagnères-de-Iachon, Argeles-(azost etc.). Enfin, un déséguilibre fondamental s'aceuse entre le secteur occidental, qui concentre lessentiel de laceroissement urbain (grandes agslomerations, forles augmentations. « villes nouvelles $\therefore$ et le secteur oriental moins bien pourve. La Garonne sépare apiomimativement ces deux zones. Ce contraste trachit l'inegale repartition des industries modernes, en particulier, la concentration des sourees d'energie entre la Garonne et le (iavo d'oloron (hỵdroélectricité, gaz) (10).

a. Les fluctuations des pelites villes de 2000 à 2010100 habitants (fig. 3) (11). - Chaque petite ville constitue un cas particulier obsissant à des faits sociaux ot économiques précis. Cependant, les petites villes pyrénéennes et sous-pyrénécnnes peuvent être regroupées en grandes catégories d'après l'évolution du nombre de leurs habitants. Ine doumine d'entre elles sont en pleine expansion. Elles ont au moins doublé entre 1936 et 1962. Les grandes industries modernes sont à l'origine de ces accroissements parfois spectaculaires. Il faut mettre a part les «villes nouvelles » du gaz de Lace : Mourenx et Artix. Mourenx-Ville Nouvelle est le type même de la ville artificielle

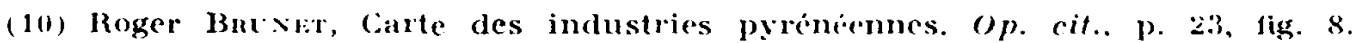

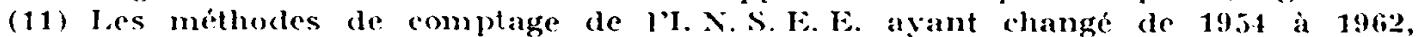
nous navons pas pu tenir compte de la colonme $k$ (population municipale agslomerce au (hef-lieu). Pour retracer lévolution des petites villes, il a donc fallu son tenir a la population communale totale (clifinition 1951. colonne c). I)ans la plupart des cas la population iparse est mulle ou tres peu nombreuse. Cependant. quelques diffeultis apparaissent dans le pays basque ou la population est assez dispersér. Ja carte chit alors etre interprétie (Hasparren, Salies-du-Salat, cte). les pourcentages divolution, compte tenu des incertitudes du recensement de 1962, n'en sont pas pour autant falsses. la periode 195-1962 itant trop courte, il a paru preferable d'itudier l'evolution depuis 1936 en tenant eompte des oscillations anterieures ot postiolieures a 1951. 


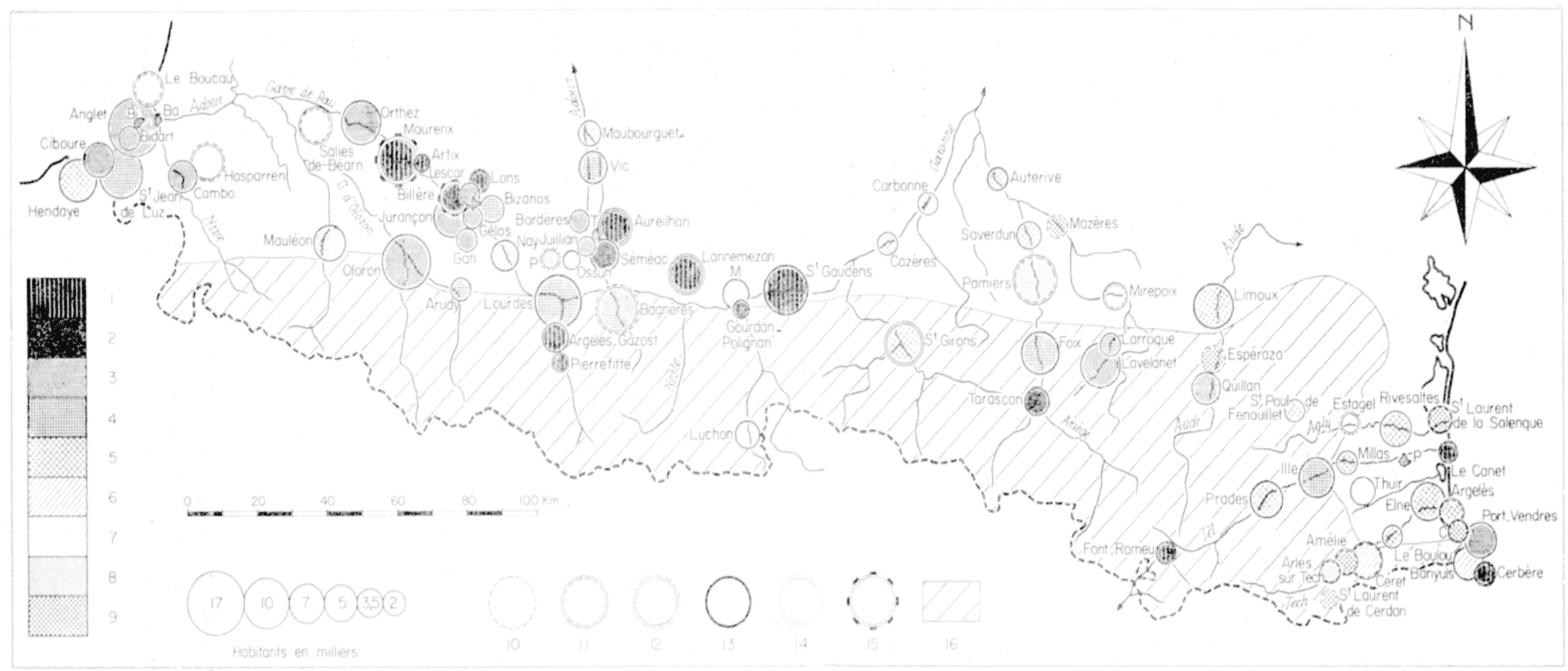

Fili, 3

Les petites villes pyrénéennes et sous-pyrénéennes $(2000-20000$ habitants) 1936-1962.

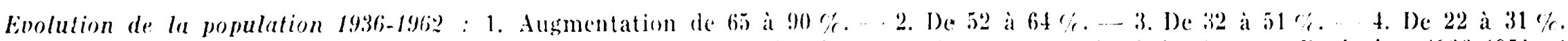

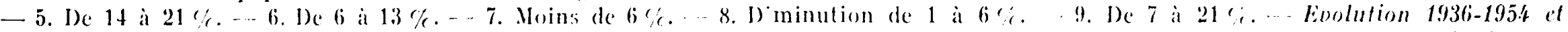
1954-1962 comparées : 10. Diminution continue 1936-1962. - 11. Diminution 1936-1954 suivie d'unc crossance 1954-1962. - 12. Croissanec

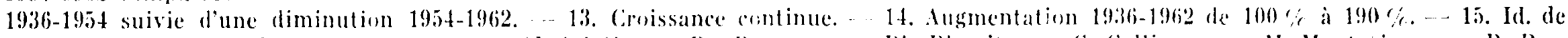
plus de $200 \%$. - 16. Zone montagneus. - Abréviations : Ba, Bayonne. .. Bi, Biarrit\%. - C. Collioure. - .l. .Hontrojeau. -. P, Parpignan. $\rightarrow$ T, 'larbes. 
par sa construction comme par la vie qu'y mènent ses habitants. En 1936, il $y$ avait 270 habitants sur l'emplacement de la future ville. Mourenx en comporte actuellement 8435 et elle n’a pas cessé de grandir. Mais son avenir est uniquement lié aux industries nées autour du gisement de Lacq. L'électrochimie et l'électrométallurgic constituent d'autres facteurs d'expansion. pierrefitte-Nestalas est passé de 911 habitants en 1936 à 2090 actuellement. Pendant la même période Argelies-(iazost a augmenté de $85 \%$ et Tarascon de $58 \%$. La construction aéronautique et les industries dérivées de l'hydroélectricité jouent un rôle identique pour les banlieues de l'agglomération tarbaise (Aureilhan 2668 lubitants en 1936. j 635 en 1962; Séméac 2752 habitants en 1936, 4480 en 1962). Il s'y ajoute deux centres touristiques en plein essor : Canet-Plage $(108 \%)$ (12) et FontRomeu $(100 \%)$. Parmi les petites villes qui ont augmenté de 20 à $50 \%$ nous trouvons les centres dinclustries traditionnelles en ple:ne activité ou récemment reconvertis : Lavelanet (51 \%), Quillan (32\%). Laroque d'Olmes $(21 \%)$. La mêne valeur d'aceroissement se retrouve pour les banlieues de pau (Jurangon 49 (i) et de Tarbes (Borderes $38 \%$, Juillan $28 \%$ ) ainsi que pour les grands centres administratifs, commerciaux et touristicues (Lourdes $35 \%$. Foix $24 \%$ ). Au-dessous de $20 \%$, les villes industrielles ne sont plus représentées que par les centres traditionnels qui ont conserve leurs anciennes structures techniques et économiques : Mauléon-Soule et Nay (17 \% r). Le Boucan. ou l'on attend la reconversion des usines siderurgiques, n'a augmenté que de $3 \%$ en 8 ans. Bagnères-de-Iuchon, qui n'est vraisemblablement plus la «Reine des Pyrénées », a très peu progressé (1936. 3591 habitants; 1954, $3666 ; 1962,3738)$.

Une dernière catégorie rassemble les villes qui ont perdu des habitants entre 1936 et 1962 . Les plus touchées $(7$ à $20 \%$ de pertes) sont Saint-Iaurent-de-la-Salenque (crise du textile). Amélie-les-Bains. Collioure (avant l’arrivée des pêcheurs algériens), Espéraza (crise de la chapellerie).

I.es fluctuations antérieures et postéricures a 1954 permettent de distinguer quatre types de progression. Ie cas le plus fréquent est celui des villes qui ont toujours augmenté avec une accélération entre 1954 et 1962 (Iannemezan, Pierrefitte, Lons, Font-Romeu, etc). Cerlaines autres, après avoir perdu des habitants entre 1936 et 1954 , se sont accrues, parfois brusquement, a la faveur d'une reconversion industrielle ou d'un aménagement touristique : Pamiers, Arles-surTech, etc. I'évolution contraire (croissance suivie d'une diminution) est exceptionnelle (Pontacq, Eslagel). Espéraza est la seule ville qui perde regulierement des habitants depuis 1936 .

Lés Pyrénées et les régions sous-pyrénéennes possèdent donc des petites villes nombreuses et relativement dynamicues. Mais elles forment moins un réseau quadrillant l'ensemble de la chaîne, qu'unc

(12) Fouto la population nest pas agglomerios an ohof-lient. 
bordure, d'autant plus active quelle so degage de la montagne. Quelle est lemprise de ces petites villes sur le milieu rural el, plus particulièrement, sur la société et l'économic montagnarle? A côté de centres équilibrés et actifs comme Saint-fiatens ou Lavelanet, quel peut être le rôle de villes sous-équipés comme Saint-(iirons ou Mircpoix? Quelle action peuvent avoir certaines villes inclustrielles. parfois implantées artificiellement (Mourenx) qui nemploient pas loujours une majorité de salariés d'origine locale? Existe-t-il un rapport liant lexpansion démographique de la petite ville a son activiti economique? Il faudrait connaitre les aracteres de lexcédent de population (âge, categorie sociale, qualification professionnelle, stabilite résidentielle). On peut, des a present disinguer deux types

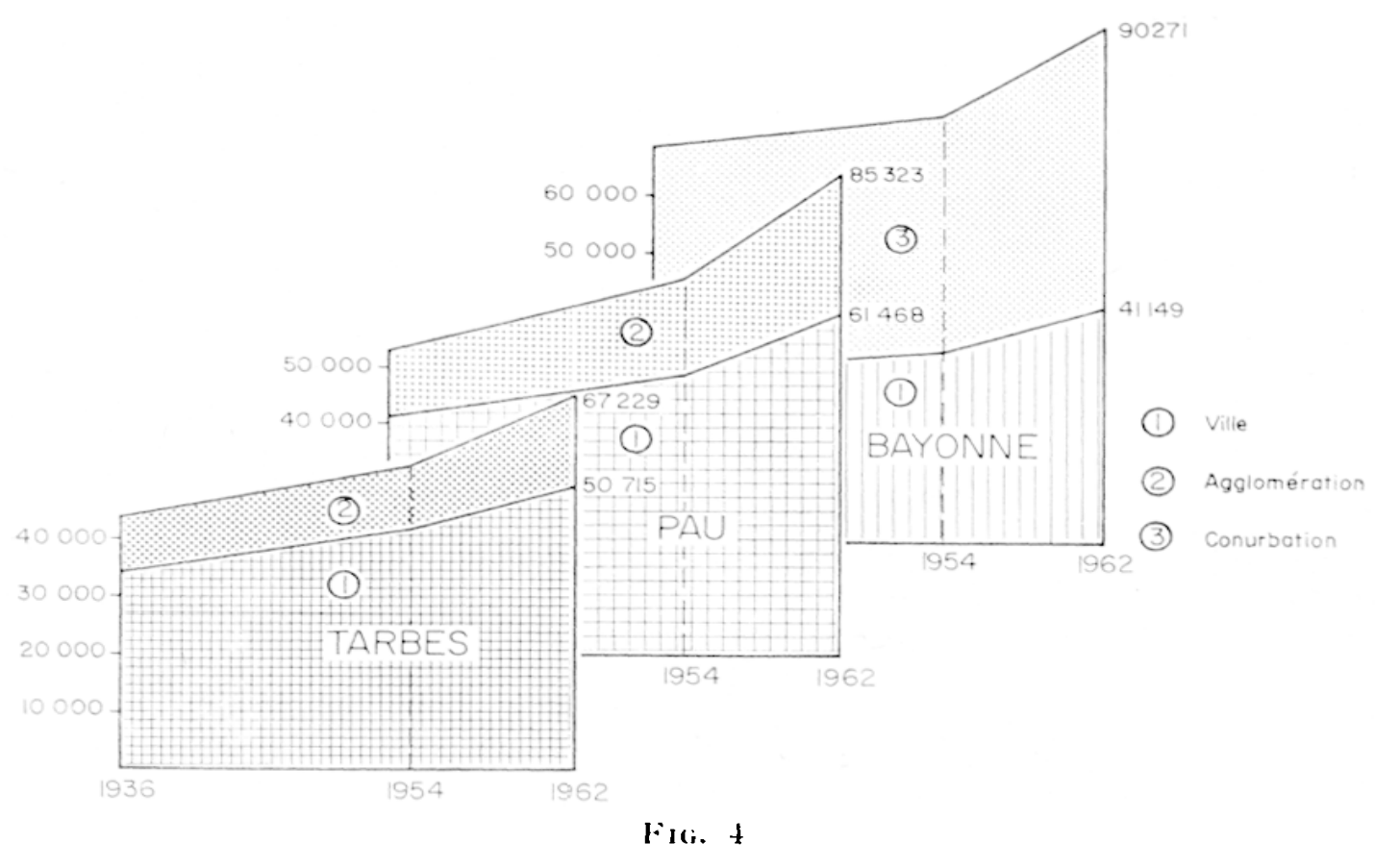

L'essor des grandes aggglomérations (1936-1962)

regionaux de petites villes. A l'Ouest de la Garonne dominent les centres industriels ot touristiques en pleine expansion, on general sans rapport direct aver la montagne. A l'Est de la Ciaronne. les petites villes paraissent mieux s’integrer a l'economic montagnarde. mais leur equilibre ne parait pas assure. En effet, la croissance de la populaton. daalleurs limitée, ne semble pass être, dans bien des cas, un signe dexpansion économicue et de irogres social. Ces deux secteurs, qui evoluent différemment, méritent un examen approfondi qui permettrait certainement de préciser les fonctions de la petife ville dans la rescau urbain moderne (13).

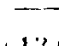

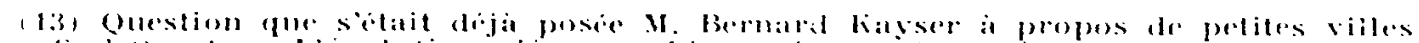

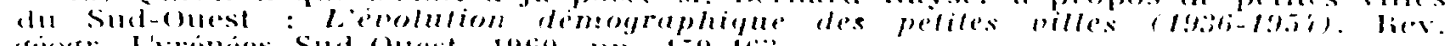

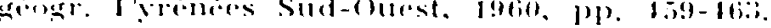


b. L'essor des grosses agglomérations (fig. 4). - Cinq villes de moyenne importance mais très étendues et très peuplées, relativement aux autres villes de la région, cristallisent l'essentiel de l'accroissement démographique par leur propre augmentation (tableau VII) et par le développement de banlieues industrielles. I.e long du littoral

Tandeal VII. - Le's principales villes sous-pyrénéennes

\begin{tabular}{|c|c|c|c|c|c|}
\hline & \multicolumn{3}{|c|}{ DEFINITION 1954} & \multirow{2}{*}{$\begin{array}{l}\text { POPULATiontotale } \\
\text { ot complee a part } \\
1962(2)\end{array}$} & \multirow{2}{*}{$\begin{array}{l}\text { EVOLE - } \\
\text { TION \% } \\
1954-1962\end{array}$} \\
\hline & 1936 & 1954 & $1962(1)$ & & \\
\hline Perpignan. .... & 72207 & 70051 & 81478 & 86156 & $+20 \%$ \\
\hline Pau. . . . . . . & 40451 & 48320 & 59879 & 61468 & $+24 \%$ \\
\hline Tarbes. . . . . & 34749 & 40242 & 49377 & 50715 & $+22 \%$ \\
\hline Bayonne.......... & 31691 & 32575 & 40219 & 41149 & $+32 \%$ \\
\hline Biarriz. . . . . & 20691 & 22922 & 24992 & 25514 & $+9 \%$ \\
\hline
\end{tabular}

Dans a tableau ot les suivants : 1962 (1) =:- definition $1951: 1962$ (2) = definition 1962.

basque s'étale une conurbation centréc sur Bayonne ef comprenant Biarritz, Anglet, Le Boucau et Saint-Pierre-d'Irube (tableau VIII). L'ensemble a augmenté de $26 \%$ entre 1936 et 1962 et comporte actuelle-

Tanteat VIII. - La comarbation bayonnaise.

\begin{tabular}{|c|c|c|c|c|c|c|}
\hline & 1936 & 1954 & 1962 (1) & $1962(2)$ & $1936-1962$ & $\begin{array}{c}\% \\
1954-1962\end{array}$ \\
\hline Bayonne. . . . . . . . . . . & 31350 & 32575 & 40219 & 41149 & & \\
\hline Biarritz. . . . . . . . . . . & 20691 & 22922 & 24992 & 25514 & & \\
\hline Anglet. . . . . . . . . . . . . & 11320 & 12603 & 16351 & 16675 & & \\
\hline Le Boucau. . . . . . . . . . . . & 5568 & 5400 & 5738 & 5875 & & \\
\hline Saint-Pierre-d'Irube. . . . . . . . & 936 & 963 & 1025 & 1056 & & \\
\hline TotaL. . . . . . . & 69865 & 74463 & 88125 & 90271 & $26 \%$ & $19 \%$ \\
\hline
\end{tabular}

ment plus de 90000 habitants. L'expansion touristique ef inclustriclle (construction aeronautique d'Angled) explique facilement cet essor. Perpignan, avee 86000 habitants, reste la deuxieme agglomération malgré l'absence de véritable banlieue industriclle. L'agglomération paloise (tablean IX) groupe 85000 habtants. En plus de la ville de Pau (61 468 hab.), elle comprend Billere, Jurangon, Bizanos, Gelos et Lons. Sa progression dépasse nettement celle des autres aggloméra- 
TanLiau IX. - L'agglomération paloise.

\begin{tabular}{|c|c|c|c|c|c|c|}
\hline & 1936 & 1954 & $1962(1)$ & $1962(2)$ & $\begin{array}{c}\text { EVOLU } \\
1936-1962\end{array}$ & $\begin{array}{l}\text { ION \% } \\
1954-1962\end{array}$ \\
\hline Pau. . . . . . . . . . . . . & 40451 & 48320 & 59879 & 61468 & 48 & 24 \\
\hline Billère. . . . . . . . . . . . . & 1930 & 3082 & 7271 & 7469 & & \\
\hline Jurançon. . . ............ & 4329 & 5561 & 6444 & 6581 & & \\
\hline Bizanos. . . . . . . . . . . . . & 2775 & 3010 & 3505 & 3586 & & \\
\hline Gelos. . . . . . . . . . . . & 2135 & 2762 & 3088 & 3150 & & \\
\hline Lons. . . . . . . . . . . . . & 1378 & 2446 & 3021 & 3069 & & \\
\hline Total banlieue. . ......... & 12547 & 16681 & 23329 & 23855 & $86 \%$ & $28 \%$ \\
\hline Totar agglomération. . ...... & 52998 & 65181 & 83208 & 85323 & $57 \%$ & $28 \%$ \\
\hline
\end{tabular}

tions $(3,3 \%$ par an entre 1936 et $1962,4,7 \%$ par an entre 1954 et 1962). La banlieue s'accroît deux fois plus vite que la ville $(86 \mathrm{c} / \mathrm{c}$ contre $48 \%$ de 1936 à 1962). Lagglomération tarbaise (tableau $\mathrm{X}$ ) : Tarbes, Aureilhan, Séméac, Bordères sur Echez, Juillan, Ibos) est

'Tableat X. -. L'agglomération tarbatse.

\begin{tabular}{|c|c|c|c|c|c|c|}
\hline \multirow[b]{3}{*}{ Tarbes. . ........ } & \multirow{3}{*}{$\begin{array}{c}1936 \\
34749\end{array}$} & \multirow{3}{*}{$\frac{1954}{40242}$} & \multirow{3}{*}{$\begin{array}{c}1962(1) \\
49377\end{array}$} & \multirow{3}{*}{$\begin{array}{l}1962(2) \\
50715\end{array}$} & \multicolumn{2}{|c|}{ EVOLUTION \% } \\
\hline & & & & & $1936-1962$ & $1954-1962$ \\
\hline & & & & & 42 & 23 \\
\hline Aureilhan. . . . . & 2668 & 4076 & 5635 & 5835 & & \\
\hline Séméac. . . ..... & 2752 & 3579 & 4480 & 4591 & & \\
\hline Bordères. . . ..... & 1686 & 2231 & 2335 & 2396 & & \\
\hline Juillian........ & 1406 & 1633 & 1940 & 1991 & & \\
\hline Ibos. . . . . . . & 1252 & 1520 & 1623 & 1701 & & \\
\hline Banlieue. . ..... & 9764 & 13039 & 16013 & 16514 & 64 & 23 \\
\hline Agglomération. .... & 44513 & 53281 & 65390 & 67229 & 46 & 23 \\
\hline
\end{tabular}

beaucoup moins peuplece (67 000 habitants) et elle n'a prosersse que de 46 ce entre 1936 et 1962 contre 57 : pour le groupe palois.

les grands foyers industriels, commorciaux et touristiques des régions sous-pyrénénnes participent peu à la vie de la montagne. Il semble meme qu'ils nattirent pas beaucoup la population pyrénéenne qui prefere emigrer vers l'aris, Bordeatux ou Toulouse. Ia 
role des petites villes ne parait pas trés différent. Mais toutes ces villes ne pourraientelles aider à la relance de l'économie montagnarde? On ne peut, pour le moment, que poser la question car il semble que les seuls centres « urbains » qui participent entièrement a la vie montagnarde soient les bourgs et les villages-centres, justement en pleine lécadence. L'exode va done continuer, allant, dans certains secteurs, jusqu'à l'abandon total. Cela ne signifie pas que la montagne ne soit plus exploitable. Seul, le systeme traditionnel de mise en valeur est en cause. Particls et fractionnes les aménagements proposés sont à peine des palliatifs qui, en général, n’intéressent pas les villes (14). La ville, souree de renouveau dans les Alpes du Nö̈d, intervient peu ici. D'ailleurs, mis a part quelques rares centres industriels et touristiques, la chaine pyrénéenne ne possède aucune véritable ville, même petite. Celles-ci ne se rencontrent que sur sa borclure, ou leur essor ne profite guère à la montagne.

Georges Bertrand.

\section{Solnces}

Recensement de 1962. Population de la France. Départements, Arrondissements, Cantons, Communes. Ministère de l'Intérieur. I.N.S.E.E.

premiers résullats généroux du recensement de 1969. Résullats pui departement et regions de programme (résullats provisoires). Bulletin hebrlomadaire de statistiques, I. N.S. E. E.. Supplément au n" 7.00 du samedi. 3 novembre 1962 (La Documentalion fransaise).

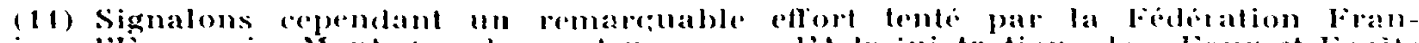

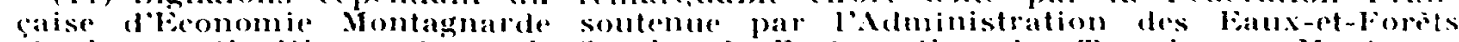

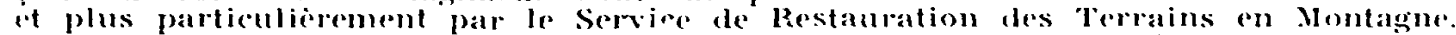
phusieurs enquetes bien documenties, utilisant des travalux geographigues, ent

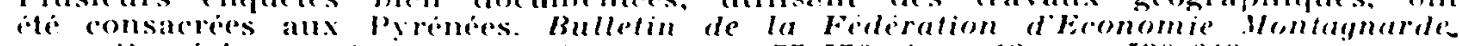

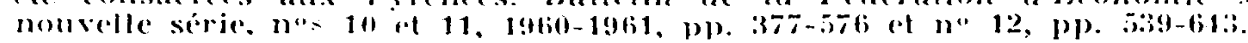

